\title{
Knockdown of toll-like receptor 4 inhibits human NSCLC cancer cell growth and inflammatory cytokine secretion in vitro and in vivo
}

\author{
DAN LI ${ }^{1}$, YINGLI JIN ${ }^{2}$, YING SUN ${ }^{1}$, JING LEI $^{1}$ and CHAOYING LIU ${ }^{1}$ \\ ${ }^{1}$ Department of Respiratory Medicine, The First Affiliated Hospital of Jilin University, ${ }^{2}$ Department of Pharmacology, \\ College of Basic Medical Science, Jilin University, Changchun, Jilin, P.R. China
}

Received March 12, 2014; Accepted May 14, 2014

DOI: $10.3892 /$ ijo.2014.2479

\begin{abstract}
Toll-like receptor 4 (TLR4)-mediated signaling has been implicated in tumor cell invasion, survival and metastasis in several types of cancers. However, the expression of TLR4 in patients with non-small cell lung cancer (NSCLC) and its biological function in the development and progression of NSCLC have not been elucidated to date. Here, we sought to characterize the expression of TLR4 in patients with NSCLC and to investigate the biological roles of TLR4 in lung metastasis, cell invasion and survival. In this study, we found that TLR4 expression was elevated in most patients with NSCLC, and its expression levels correlated with key pathological characteristics, including tumor differentiation, stage and metastasis. Our data also showed that downregulation of TLR4 expression using an RNA silencing approach in A549 tumor cells significantly suppressed cell proliferation, cell migration and cell invasion, and induced tumor apoptosis in vitro, and suppressed tumor growth in vivo. In addition, we also found that downregulation of TLR4 expression significantly decreased cell TNF- $\alpha$ and IL- 6 levels. Furthermore, we found that knockdown of TLR4 was able to significantly suppress constitutive phosphorylation of Akt and PI3K, which may contribute to the inhibition of tumor growth. These data suggest that TLR4 plays an important role in tumorigenic properties of human NSCLC, and that RNA interference-directed targeting of TLR4 could be used as a potential anticancer therapeutic target in NSCLC.
\end{abstract}

Correspondence to: Professor Chaoying Liu, Department of Respiratory Medicine, The First Affiliated Hospital of Jilin University, Changchun, Jilin, P.R. China

E-mail: liuchaoying308@sina.com

Key words: non-small cell lung cancer, toll-like receptor 4, RNA silencing, cytokines

\section{Introduction}

Non-small cell lung cancer (NSCLC) accounts for approximately $85 \%$ of all lung cancers diagnosed (1), with less than $15 \%$ of patients surviving beyond 5 years due to lack of early diagnosis and effective treatment methods (2,3). Despite the advances in multimodal neoadjuvant chemotherapy and radiotherapy in treatment NSCLC, the outcome remains unsatisfactory because these therapies are toxic and almost never curative of metastatic NSCLC (4). Thus, this has highlighted the necessity for new therapeutic modalities, particularly for patients whose disease does not respond to conventional therapy.

Human toll-like receptors (TLRs) were first identified in mammalian immune cells, and belong to type I transmembrane proteins family consisting of an extracellular domain with a leucine-rich repeat region and an intracellular domain homologous to that of the human interleukin (IL)-1 receptor (5). TLRs have a powerful capacity to innate immune responses (6) through recognition of pathogen-associated molecular patterns (PAMP) expressed by viruses and bacteria, or host-derived PAMPs (7). Until now, 11 mammalian TLRs have been identified and found to be involved in the recognition of PAMPs $(8,9)$. TLR-4 is an important member of type I transmembrane proteins family. Recently, growing evidence has shown TLR4 in various tumors (10-12), including head and neck, lung, gastrointestinal, liver, pancreatic, skin, breast, ovarian, cervical and prostate cancer (13). Although the TLR-4 profile varies in different tumor cells, current evidence indicates that the expression of TLR-4 and signaling cascade are involved in tumor growth, progression and invasion (14). For example, TLR-4 and signaling increased COX-2 and PGE2 signaling and early colorectal carcinogenesis, inhibited apoptosis and promoted angiogenesis (15). TLR4 increases Nanog gene expression, which induces liver oncogenesis (16). TLR4-mediated cancer growth involved in breast tumor progression and downregulation of TLR4 prevented breast cancer progression and survival (17). TLR4 expressed on human lung cancer cells is functionally active, and may play important roles in promoting immune escape of human lung cancer cells by inducing immunosuppressive cytokines and apoptosis resistance (18). TLR4 acts as a functional receptor in the pre-metastatic phase in pulmonary metastasis (19). These studies implied that TLR4 are widely expressed on human 
tumor cells and may play important roles in the initiation and progression of cancer. However, to our knowledge, the effect of TLR4 expression on tumorigenicity in lung cancer in vivo and in vitro has been insufficiently reported $(18,20)$, not fully clarifying the of TLR4 in NSCLC (20).

Therefore, in the present study we investigated the expression of TLR4 in NSCLC, and analyzed its association with the occurrence and development of NSCLC. In addition, we also developed a plasmid vector expressing siRNA-directed against TLR4 to investigate the key roles in tumor growth in vitro and in vivo for NSCLC.

\section{Materials and methods}

Tissue samples and clinical information. All patients gave written informed consent to participate in the study. This study was approved by the Ethics Committee of Jilin University, Changchun, Jilin Province, China.

Surgical tissue samples of human tumors and tumor-free tissues were obtained from patients undergoing surgical resection at the First Affiliated Hospital of Jilin University (Changchun, Jilin, China) from March, 2009 to July, 2013 after consent was obtained from the patients. All samples were immediately frozen in liquid nitrogen and stored at $-80^{\circ} \mathrm{C}$ until use. Tumor-free tissues were excised at least $5 \mathrm{~cm}$ away from the tumor border. Fifty tumor tissue and tumor-free tissues of NSCLC were classified into adenocarcinoma (24/50) and squamous cell carcinoma (26/50) according to the criteria of WHO (1997). Clinical information of all patients was collected including age, gender, smoking, tumor histological type, lymph node metastasis, TNM stage and differentiation. None of the patients received any prior radiochemotherapy. Clinical information of study subject is given in Table I.

Immunohistochemistry. For immunohistological analyses, tissue specimens were fixed in $10 \%$ formalin buffer at $\mathrm{pH} 7.0$ for $24 \mathrm{~h}$ and paraffin embedded. Lung tissue specimens were embedded in paraffin and cut into $3-\mu \mathrm{m}$ sections for use in immunohistochemistry. Sections were dewaxed in xylene, rehydrated in alcohol in descending percentage, and blocked for endogenous peroxidase and avidin/biotin activities with $3 \%$ bovine serum albumin in $0.01 \mathrm{M}$ phosphate-buffered saline (PBS, pH 7.2). Sections were incubated with mouse monoclonal antibody against human TLR4 (Santa Cruz Biotechnology, Santa Cruz, CA, USA) at a dilution of 1:1,500 overnight at $4^{\circ} \mathrm{C}$. Samples were then washed in 0.01 M PBS (pH 7.2) for $5 \mathrm{~min}$ before incubation with biotin-labelled rabbit anti-mouse antibody (1:1,000; ZSGB-Bio, Beijing, China) for $2 \mathrm{~h}$ at $37^{\circ} \mathrm{C}$. After washing with PBS three times, the immunostain was visualized with a streptavidin-peroxidase reaction system (Wuhan Boster Biological Technology Ltd, Wuhan, China), and developed with diaminobenzidine hydrogen peroxide (Wuhan Boster Biological Technology Ltd).

The intensity of the staining was graded as: 0 , if no immunoreactive cells were observed (negative); $1+$, if the proportion of immunoreactive cells was $<25 \% ; 2+$, if the proportion of immunoreactive cells was $25-75 \%$; and $3+$, if the proportion of immunoreactive cells was $>75 \%$. Values of 0 and 1 were considered to indicate negative staining, and 2 and 3 were considered to indicate positive staining.
Table I. The correlation of TLR4 expression with clinical pathologic features of NSCLC.

\begin{tabular}{|c|c|c|c|}
\hline Clinical factor & Positive & Negative & P-value \\
\hline Age & & & 0.489 \\
\hline$<60(\mathrm{n}=29)$ & 18 & 11 & \\
\hline$\geq 60(n=21)$ & 15 & 6 & \\
\hline Gender & & & 0.356 \\
\hline $\operatorname{Man}(n=26)$ & 17 & 9 & \\
\hline Women $(n=24)$ & 16 & 8 & \\
\hline Smoke & & & 0.413 \\
\hline No $(n=31)$ & 20 & 11 & \\
\hline Yes $(n=19)$ & 13 & 6 & \\
\hline Metastasis & & & $<0.01$ \\
\hline No $(n=20)$ & 8 & 12 & \\
\hline $\mathrm{N} 1-\mathrm{N} 2(\mathrm{n}=25)$ & 10 & 15 & \\
\hline $\operatorname{M1}(n=5)$ & 5 & 0 & \\
\hline Tumor differentiation & & & $<0.01$ \\
\hline Well $(n=8)$ & 1 & 7 & \\
\hline Moderate $(n=26)$ & 18 & 8 & \\
\hline Poor $(n=16)$ & 14 & 2 & \\
\hline TNM stage & & & $<0.05$ \\
\hline $\mathrm{I}-\mathrm{II}(\mathrm{n}=25)$ & 8 & 17 & \\
\hline III-IV (n=25) & 17 & 8 & \\
\hline Histological type & & & 0.347 \\
\hline Adenocarcinoma $(n=24)$ & 15 & 9 & \\
\hline $\begin{array}{l}\text { Squamous cell } \\
\text { carcinoma }(n=26)\end{array}$ & 18 & 8 & \\
\hline
\end{tabular}

Cell culture. The human non-small cell lung cancer cell line A549 was purchased from Cell Bank of Type Culture Collection of Chinese Academy of Sciences, Shanghai Institute of Cell Biology, Chinese Academy of Sciences (Shanghai, China). A549 cells were cultured in RPMI-1640 medium (Invitrogen, USA) supplemented with $10 \%$ fetal bovine serum (FBS, Gibco, USA), cells were maintained at $37^{\circ} \mathrm{C}$ in a humidified atmosphere containing $5 \% \mathrm{CO}_{2}$.

Construction of expressing plasmids. To inhibit the expression of TLR4, two short hairpin RNA (shRNA) targeting the TLR4 transcript were designed and synthesized and annealed. The synthesized oligonucleotides contain specific target sequence, a loop, the reverse complement of the target sequence, a stop codon for U6 promoter and two sticky ends. The target sequence in the oligonucleotide for suppressing TLR-4 was as followed: the siRNA1 sequence is: AACTTGTATTCAAGGTC TGGC (sense); the siRNA2 sequence is: AACTCCCTCCAGG TTCTTGAT (sense). The scramble sequence is: AATTCTCCG AACGTGTCACGT (sense). The empty vector (pCDNA-CMV) and scramble sequence had been tested in multiple cell lines and did not demonstrate any toxicity to cells as demonstrated by MTT assay after transfection, and had no effect on the expression of housekeeping genes, GAPDH or $\beta$-actin. The siRNA1, siRNA2 and scramble sequence were cloned into 
expressing plasmid pCDNA-CMV, named as p-siRNA1, p-siRNA2 and p-scramble, respectively.

A549 cells were transiently transfected with empty vector (pCDNA-CMV), p-siRNA1, p-siRNA2 and p-scramble plasmid using Lipofectamine ${ }^{\mathrm{TM}} 2000$ reagent (Invitrogen) according to the manufacturer's instructions, respectively. After $72 \mathrm{~h}$ of transfection, cells were collected and used for cell proliferation assays, cell apoptosis assays, western blot analysis, real-time quantitative PCR analysis, Matrigel invasion assay, caspase activity assay and cell inflammatory cytokines assay. Empty plasmid (pCDNA-CMV) acted as control group in the assays.

Quantitative PCR. Total RNA was isolated from A549 cell lines and NSCLCs tissue using RNeasy mini kit (Qiagen, Valencia, CA, USA) according to the manufacturer's instructions. RNA was prepared using the. RNA was reverse transcribed into cDNA by a Primescript ${ }^{\mathrm{TM}}$ RT reagent kit base on the manufacturer's protocols (Takara, Dalin, China). Quantitative real-time polymerase chain reaction (qPCR) assays were performed with SYBR-Green Real-Time PCR Master Mix (Toyobo, Osaka, Japan) and amplification equipment using specific primers: TRL4 forward, 5'-CGAGGA AGAGAAGACACCAGT-3' and reverse, 5'-CATCATCCTCA CTGCTTCTGT-3'; GAPDH forward, 5'-TGTGGGCATCAAT GGATTTGG-3' and reverse, 5'-ACACCATGTATTCCGGGT CAAT-3'. The PCR conditions were as follows: a pre-denaturing at $95^{\circ} \mathrm{C}$ for $5 \mathrm{~min}$, followed by 40 cycles of denaturation at $95^{\circ} \mathrm{C}$ for $20 \mathrm{sec}$, annealing/extension at $55^{\circ} \mathrm{C}$ for $20 \mathrm{sec}$, final extension $72^{\circ} \mathrm{C}$ for $10 \mathrm{~min}$. The amplification specificity was checked by melting curve analysis. The $2^{-\Delta \Delta \mathrm{CT}}$ method (21) was used to calculate the relative abundance of target gene expression generated by Boineer Exicycler ${ }^{\mathrm{TM}}$ analysis software (Bioneer Corp., Daejeon, Korea). The expression of TLR4 were determined by normalization of the threshold cycle $(\mathrm{Ct})$ of these genes to that of the control GAPDH. Each sample was run in triplicates.

Western blot analysis. After $72 \mathrm{~h}$ of transfection, A549 cells were washed twice with PBS and lysed in radio immune precipitation assay buffer for $30 \mathrm{~min}$ on ice. Cell lysates were clarified by centrifugation at $10,000 \mathrm{x}$ g for $15 \mathrm{~min}$, and protein concentrations were determined using the Bradford reagent (Sigma Chemical Co., St. Louis, MO, USA). Lysates were separated on 8 or $15 \%$ SDS-PAGE; proteins were transferred to Immobilon membrane (Millipore, Bedford, MA, USA) immunoblotted with specific primary antibodies and incubated with corresponding horseradish peroxidase- conjugated secondary antibody. Protein bands were visualized with enhanced chemiluminescence reagent (ECL, Amersham, GE Healthcare, Velizy-Villacoublay, France). The primary antibodies used in the western blots were as follows: antibodies against TLR4, $\beta$-actin, MMP-2 and MMP-9 (Santa Cruz Biotechnology); Akt, phosphorylated (p-) Akt, PI3K and p-PI3K (Sigma Chemical Co.). Secondary Abs used for immunodetection were: HRP-conjugated goat anti-mouse IgG and goat anti-rabbit IgG (Amersham Biosciences, Uppsala, Sweden).

MTT assay. The cell density of A549 cells was adjusted to $5 \times 10^{4} / \mathrm{ml}$, and cells were added to a 96-well plate (100 $\mu 1 /$ well). In the blank controls, $100 \mu \mathrm{l}$ of medium alone was added. At
$24 \mathrm{~h}$ after culture, cells were transfected with the indicated plasmid. At $72 \mathrm{~h}$ after culture, $20 \mu \mathrm{l}$ of 3-(4,5-dimethylthiazol2-yl)-2,5-diphenyltetrazolium bromide (MTT,5 mg/ml) (Sigma Chemical Co.) was added to each well followed by incubation at $37^{\circ} \mathrm{C}$ for $48 \mathrm{~h}$. Then, centrifugation was performed at $2,000 \mathrm{x} \mathrm{g}$ for $10 \mathrm{~min}$. The supernatant was removed, and $200 \mu \mathrm{l}$ of DMSO was added to each well followed by shaking for $10 \mathrm{~min}$. Absorbance was measured at $570 \mathrm{~nm}$ test wavelength with an ELISA multi-well spectrophotometer (Molecular Devices Corp., Sunnyvale, CA, USA). The mean proliferation of cells without any treatment was expressed as $100 \%$. Growth inhibition was calculated with the following formula (22): inhibition rate $(\%)=[1-($ average absorbance of experimental group/average absorbance of blank control group)] x 100\%.

Apoptosis analysis. A549 cells were cultured in 6-well plates in RPMI-1640 medium containing 10\% FBS and were treated with indicated plasmid for $72 \mathrm{~h}$. The cover slips were washed three times with PBS (pH 7.2), then cells were stained with $100 \mu \mathrm{g} / \mathrm{ml}$ acridine orange (AO) and $100 \mu \mathrm{g} / \mathrm{ml}$ ethidium bromide (EB) for $1 \mathrm{~min}$. Cells were observed under a fluorescence microscope (Olympus, Tokyo, Japan). At least 200 cells were counted and the percentage of apoptotic cells was determined. We also detected caspase- 3 and caspase- 8 activity by ELISA as an additional indicator of apoptosis.

Caspase activity assay. The activity of caspase- 3 and caspase- 8 was measured using caspases colorimetric protease assay kits (Millipore Corporation, Billerica, MA, USA) according to the manufacturer's instructions. In brief, A549 cells were treated with indicated plasmid for $24 \mathrm{~h}$. After treatment, cells were washed twice with ice-cold PBS (pH 7.2) and harvested by centrifugation at $700 \mathrm{xg}$ for $10 \mathrm{~min}$. The cell pellets were then lysed in $150 \mu \mathrm{l}$ buffer provided in the kit. Protein concentrations of lysates were determined using the Lowry method (23). Then, an aliquot of lysates $(80 \mu \mathrm{l})$ was incubated with $10 \mu \mathrm{l}$ substrate of each caspase at $37^{\circ} \mathrm{C}$ for $2 \mathrm{~h}$. Samples were analyzed at $405 \mathrm{~nm}$ in a microplate reader (Thermo Fisher Scientific Inc., Waltham, MA, USA). The relative caspase activity of the control group was taken as 100. Each sample was run in triplicates.

Migration assay. Cell migration was determined using a scratch assay as previous described (24). In brief, the transfected cell lines were seeded on a 24-well plate and allowed to reach confluence. After scratching the bottom of the well with a pipette tip, then the monolayer of cells was washed three times with PBS (pH 7.2) to remove the detached cells. The remaining adherent cells were incubated in RPMI-1640 medium containing $1 \% \mathrm{FBS}$ for $24 \mathrm{~h}$; this medium was then replaced with RPMI-1640 medium containing 10\% FBS. After $48 \mathrm{~h}$, cell migration was evaluated using bright-field microscopy. The experiments were performed in triplicate.

Invasion assay. Invasion assay were performed using BD BioCoat ${ }^{\mathrm{TM}}$ Matrigel invasion chambers (Becton-Dickinson Labware, Bedford, MA, USA) according to the manufacturer's instructions. RPMI-1640 was added to the interior of the bottom and top chamber of inserts and allowed to hydrate for $2 \mathrm{~h}$ at $37^{\circ} \mathrm{C}$ with $5 \% \mathrm{CO}_{2}$. Albumin at $100 \mathrm{ng} / \mathrm{ml}$ was added to the bottom chamber. Next, $5 \times 10^{4}$ A549 cells transfected with 
indicated plasmids were added to the top chamber of inserts and incubated at $37^{\circ} \mathrm{C}$ and $5 \% \mathrm{CO}_{2}$ for $24 \mathrm{~h}$. After incubation, cells at the bottom surface of the insert were fixed with purity methanol for $2 \mathrm{~min}$, stained for $2 \mathrm{~min}$ in $1 \%$ toluidine blue supplemented with $1 \%$ borax (all from Sigma Chemical Co.), and rinsed twice with deionized water (distilled $\mathrm{H}_{2} \mathrm{O}$ ). The cells that had invaded to the lower side of the filter were observed under a Nikon phase-contrast microscope and counted in $>10$ fields of view at $\times 200$ magnification. The assay was done in triplicate. We also detected MMP-2 and MMP-9 by western blot analysis as an additional indicator of migration and invasion.

Human inflammatory cytokine assay. IL-6, IL-8 and TNF- $\alpha$ presence in the supernatant of transfected cells were detected according to the instruction of human inflammatory cytokine kit (BD ${ }^{\mathrm{TM}}$ Cytometric Bead Array, Becton-Dickinson Labware). FACScan flow cytometer (BD) was used to analyze samples.

Tumor xenograft assay. About 6-8 weeks old female BALB mice were obtained from the Institute of Laboratory Animal Science, Jilin University (Changchun, China), and were maintained under specific pathogen-free conditions and provided with food and water ad libitum. All animal experiments were performed in accordance with institutional guidelines, following a protocol approved by the Ethics Committees of the Disease Model Research Center, Jilin University (Changchun, China).

All the animals were fed with a normal pellet diet one week prior to the experimentation. A549 cells in exponential growth phase were harvested and single-cell suspensions ( $2 \times 10^{6}$ cells in $\left.100 \mu 1 \mathrm{PBS}\right)$ were injected subcutaneously (s.c.) into the right dorsal flank of nude mice. Tumor size was measured every 2 to 3 days, and tumor volume calculated as $0.5236 \mathrm{x}$ width $^{2} \mathrm{x}$ length. When tumors grew to an average volume of $75 \mathrm{~mm}^{3}$, mice were randomly divided into siRNA, scramble group and control group ( $\mathrm{n}=10$ in each group), and inoculated with $30 \mu \mathrm{g} / 50 \mu \mathrm{l}$ per mouse via i.t. injection of indicated plasmids one time a week for 21 days, respectively. Mice were sacrificed 7 days after the final plasmid injection. Tumor tissue was excised, measured volume and weighed. Some of the tissue was snap-frozen immediately for immunoblotting.

Statistical analysis. Statistical analyses were undertaken using the SPSS $^{\circledR}$ statistical package, version 16.0 (SPSS Inc., Chicago, IL, USA) and the GraphPad Prism version 5.01 (GraphPad Software, San Diego, CA, USA) for Windows ${ }^{\circledR}$. All data are expressed as mean \pm SD. Statistical analysis between two samples was performed using Student's t-test. Statistical comparison of more than two groups was performed using one-way ANOVA followed by a Tukey's post hoc test. Pearson's correlation coefficients were used to determine whether two prognosis related factors were correlated to each other over all cases. $\mathrm{P}<0.05$ was considered significant.

\section{Results}

TLR4 is upregulated in NSCLC and correlates with clinical features of patients with NSCLC. To identify the potential roles of TLR4 in the development and progression of NSCLC, we assessed its mRNA expression level and protein expression

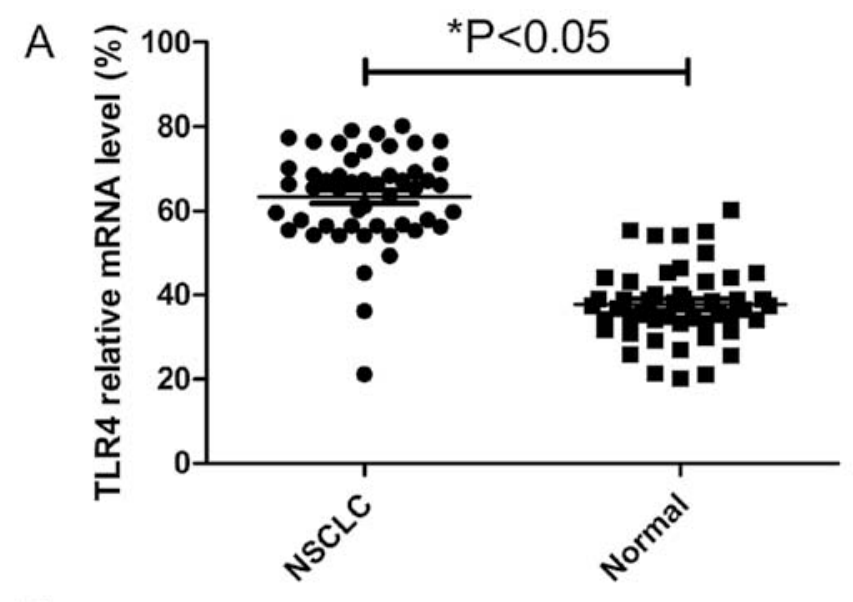

$B$

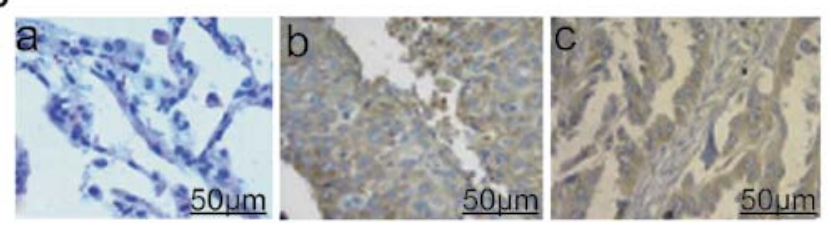

Figure 1. Levels of TLR4 are increased in NSCLC clinical samples. (A) Shown are quantitative real-time PCR results of relative expression level of TLR4 in 50 cases of NSCLC and cancer-free samples. The mRNA expression level of TLR4 was quantified as an internal standard and used to normalize the level of GAPDH from the same sample. (B) Shown are immunohistochemical results of TLR4 expression in NSCLC and matched cancer-free lung tissues: a, cancer-free lung tissue; b, adenocarcinoma; c, squamous cell carcinoma; $* \mathrm{P}<0.05$ vs. cancer-free lung tissue.

level in 50 pairs of matched lung tissue samples by real-time polymerase chain reaction (qPCR) and immunohistochemistry, respectively. Real-time polymerase chain reaction (qPCR) assay showed that mRNA expression levels of TLR-4 were significantly higher in NSCLC tumors compared with their normal lung counterparts $(\mathrm{P}<0.05$, Fig. 1A). At protein level, elevated levels of TLR4 protein were found in NSCLC tumors compared with the paired normal tissues from the same patients as shown by immunochemical staining (Fig. 1B).

Testing the association between TLR4 immunostaining with the clinicopathological parameters of the patients with NSCLC showed no significant differences with regard to patient gender, age and smoking history. The TLR4-positive tumors were of larger size, were poorly differentiated, had a higher TNM stage and were more likely to have metastasis than the TLR4-negative tumors $(\mathrm{P}<0.05$, Table I).

Knockdown of TLR4 gene using siRNA in human A549 lung cancer cells. To study the biological role of TLR4 in NSCLC progression, we first constructed pcDNA3-CMV vectors expressing two small hairpin siRNA oligonucleotides targeting TLR4 (GenBank: NM-138554.3) to selectively reduce TLR4 gene expression in A549 cells, then the vectors expressing TLR4 siRNA or scramble siRNA were transfected into human lung cancer A549 cells. To determine the effect of siRNA on the endogenous expression of TLR4, the mRNA and protein levels of TLR4 were analyzed with real-time RT-PCR and western blot analysis, respectively. As shown in Fig. 2A, at mRNA level, there were different reductions in siRNA1, siRNA2 transfected cells and the decreased expression of TLR4 at mRNA levels 

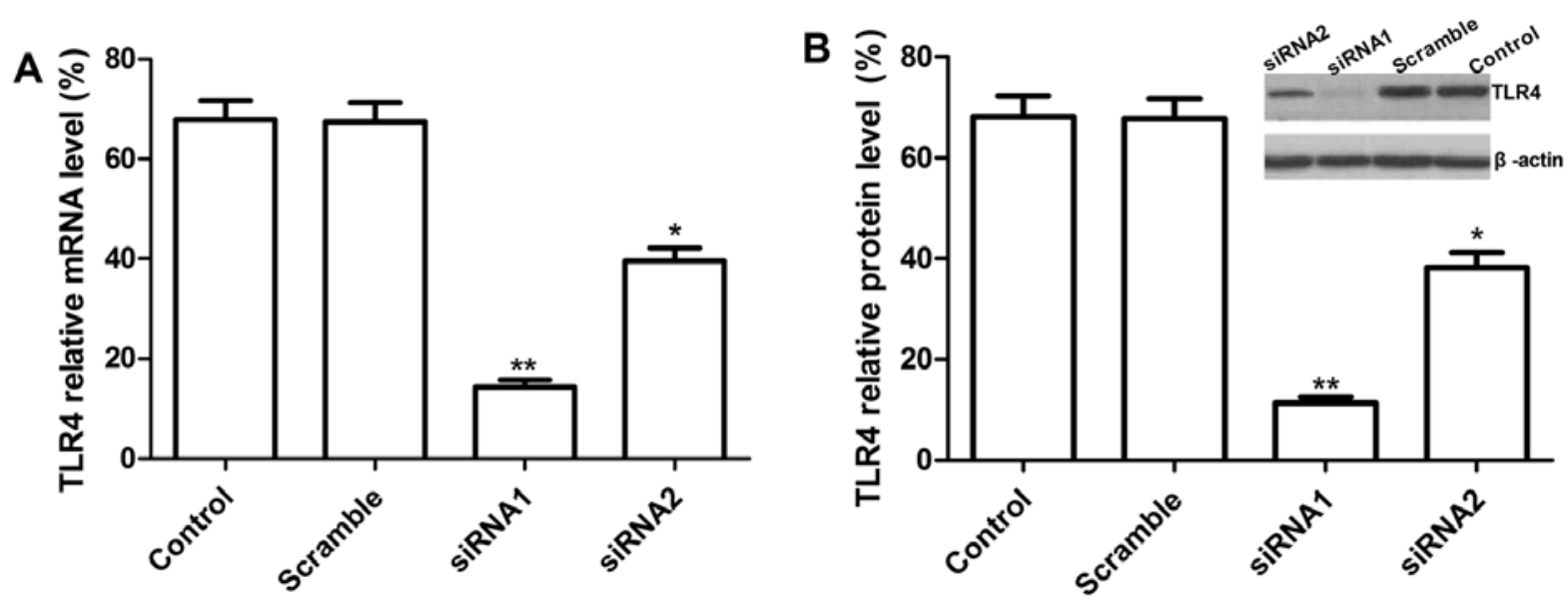

Figure 2. Knockdown of TLR4 inhibits the TLR4 expression in A549 cancer cells. (A) Quantitative real-time PCR analysis of TLR4 $72 \mathrm{~h}$ after various plasmid transfection. (B) Western blot analysis of TLR4 $72 \mathrm{~h}$ after various plasmid transfection and RNAi silencing; ${ }^{* *} \mathrm{P}<0.01 \mathrm{vs}$. control.
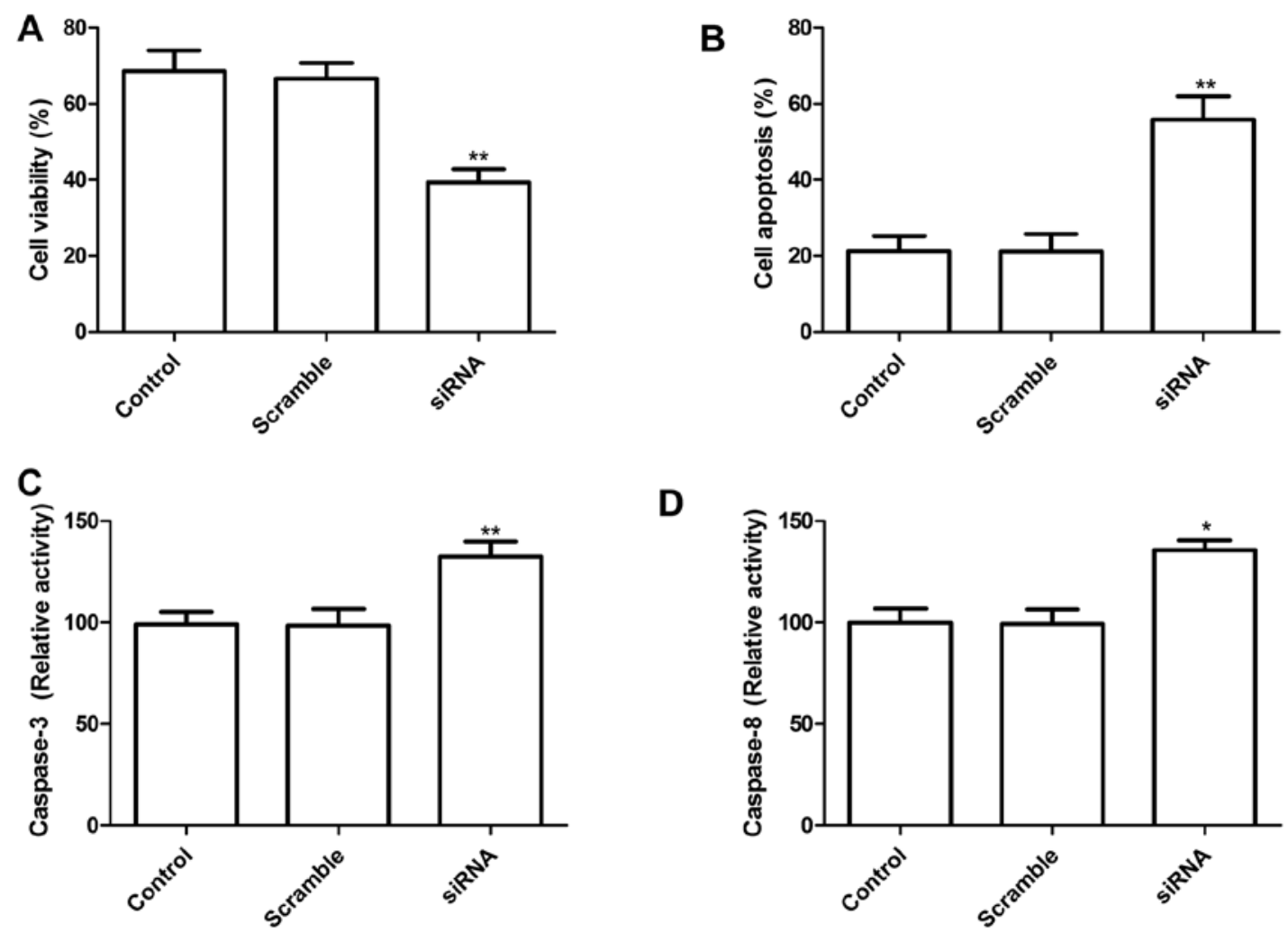

Figure 3. Knockdown of TLR4 inhibits cancer cell proliferation and induced cell apoptosis in vitro. (A) Cell proliferation and (B) cell apoptosis of A549 cells was determined after treated with indicated plasmid. (C) Caspase-3 and (D) caspase-8 activity of A549 cells was determined after treated with indicated plasmid ${ }^{*} \mathrm{P}<0.05,{ }^{* * *} \mathrm{P}<0.01$ vs. control.

for siRNA1, siRNA2 was $75.1 \pm 8.5,46.2 \pm 4.7 \%$ as compared to vector control $(\mathrm{P}<0.05)$. However, no significant difference was observed in scramble and control group (empty group) $(\mathrm{P}>0.05)$. At protein level, western blot analysis results showed that two independent target sequences siRNA1 and siRNA2 markedly decreased protein expression of TLR4 compared with the scramble and control group $(\mathrm{P}<0.05$, Fig. $2 \mathrm{~B})$ and siRNA1 had high reduction ratio as compared to siRNA2. Therefore, siRNA1 was chosen for use in subsequent functional assay.
Knockdown of TLR4 in lung cells reduces proliferation and induction of apoptosis. To determine the potential effects of siRNA-mediated TLR4 silencing on cell proliferation and survival, MTT analysis was performed $72 \mathrm{~h}$ after transfection with siRNA targeting TLR4. The results clearly show that transfection of A549 cells with siRNA targeting TLR4 significantly inhibited cell proliferation as compared to control group and scramble group $(\mathrm{P}<0.01$, Fig. $3 \mathrm{~A})$. Next, the effects of the knockdown TLR4 by siRNA on lung cancer cell apoptosis were 

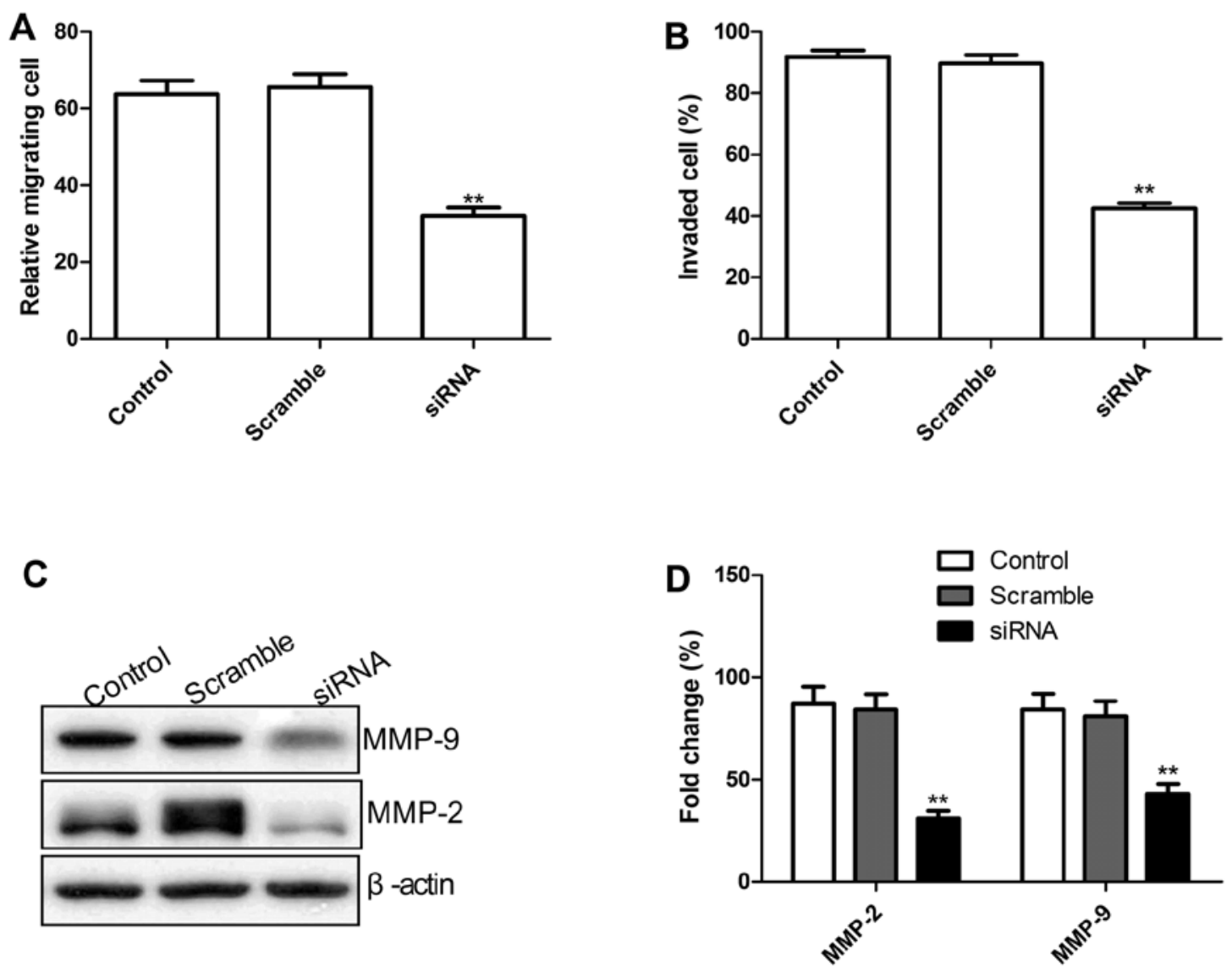

Figure 4. Knockdown of TLR4 inhibits cancer cell migration and invasion in vitro. (A) Cell migration and (B) cell invasion of A549 cells was determined after treated with indicated plasmid. (C and D) Western blot analysis was used to assess expression of MMP-2 and MMP-9 using antibodies against MMP-2 and MMP-9. $\beta$-actin was used as an internal control; ${ }^{*} \mathrm{P}<0.05,{ }^{* *} \mathrm{P}<0.01$ vs. control.

assessed. As shown in Fig. 3B, knockdown of TLR-4 induced cell apoptosis compared to control group and scramble group $(\mathrm{P}<0.01)$.

To determine the potential mechanism of cell growth inhibition in vitro, caspase- 3 and caspase- 8 activity was detected using ELISA. Caspase- 3 and caspase- 8 activity was significantly decreased in knockdown TLR4 treatment groups, compared to the controls and scramble siRNA groups $(\mathrm{P}<0.05$; Fig. $3 \mathrm{C}$ and $\mathrm{D})$. These results suggest that reducing TLR4 levels may inhibit cell proliferation and induce cell apoptosis in lung cancer cells.

Knockdown of TLR4 in lung cells inhibits cell migration and cell invasion. To ascertain the inhibitory effect of knockdown of TLR4 on lung cancer on cell motility in vitro, scratch assay was performed to investigate their effects on the migration potential of A549 cells. As shown in Fig. 4A, knockdown TLR4 by siRNA significantly reduced the migration capacity in A549 tumor cells $(\mathrm{P}<0.01)$.

To evaluate the impact of the TLR4 knockdown on invasion of human lung cancer cells A549, invasion assay using the siRNA-transfected cells was performed. Our results showed that cell invasion ability in the knockdown TLR4 group was significantly decreased compared with controls and scramble groups, when assessed after $48 \mathrm{~h}$ by the modified Boyden chamber assays (Fig. 4B).
Migration and invasion play a crucial role in tumor metastasis. To determine the potential mechanism of the TLR4 knockdown on the inhibition of cell migration and invasion in vitro, MMP-2 and MMP-9 protein expression was examined using western blots. Western blot analysis displayed a significant decrease in MMP-2, and MMP-9 proteins in the knockdown TLR4 group infected A549 cells compared to control and Scramble groups (Fig. 4C and D). Taken together, these results suggest that reduction of TLR-4 on the inhibitory effect of metastasis of lung cancer was at least partially mediated by the downregulation of MMP-9 and MMP-2. These data indicate that TLR4 plays an important regulatory role in tumor cell migration and invasion.

TLR4 knockdown in lung cells inhibits TNF- $\alpha$ and IL-6. To examine the status of the TLR4-related inflammatory cytokines in the lung cell line A549 with TLR4 gene knockdown, ELISA assay was performed. Our results demonstrated that IL-6 and TNF- $\alpha$ were markedly depressed in the supernatant of silenced cells. The inhibition ration of cytokine IL-6 and TNF- $\alpha$ was $45.3 \pm 3.6$ and $46.1 \pm 3.5 \%$, respectively, when compared with vector control $(\mathrm{P}<0.05$, Fig. $5 \mathrm{~A}$ and $\mathrm{B})$, no significant difference occurred in control group and scramble group (Fig. 5A and B). These results suggested that decreased TLR4 levels in tumor cells might reduce inflammatory cytokines. 

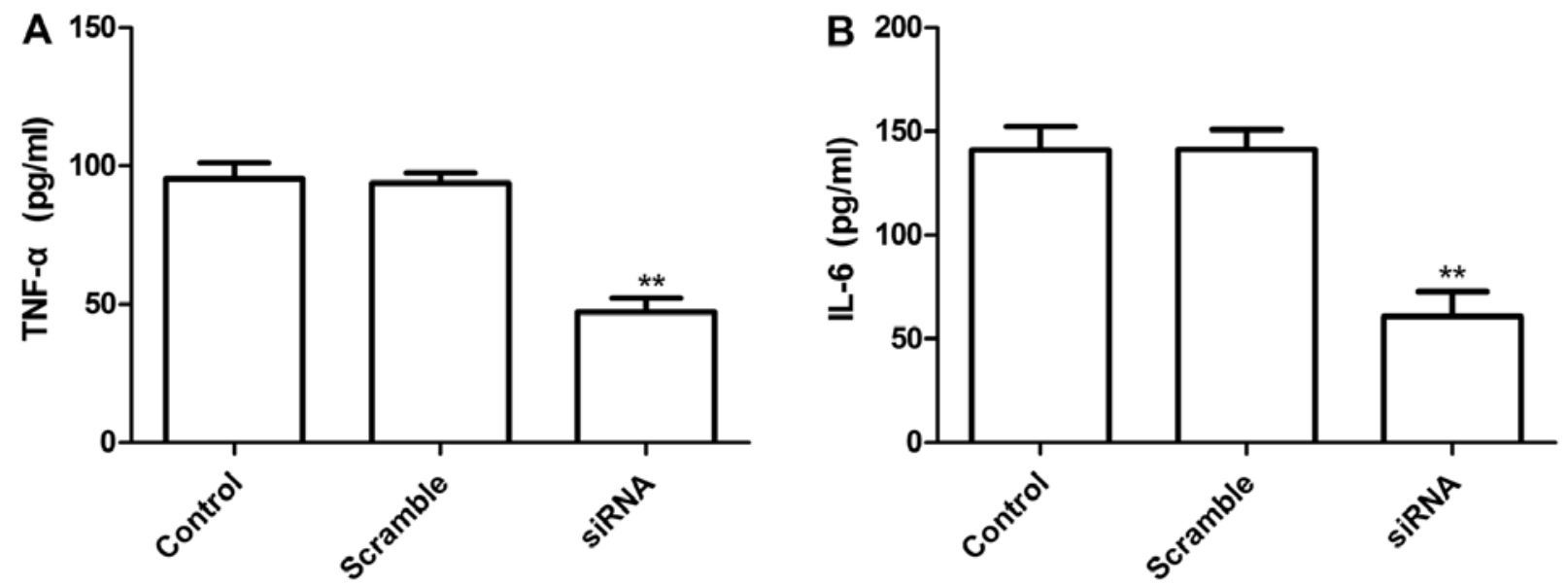

Figure 5. Knockdown of TLR4 inhibits inflammatory cytokines of A549 cells. (A) TNF- $\alpha$ and (B) IL-6 levels were measured by ELISA after treatment with indicated plasmid; ${ }^{\mathrm{P}}<0.05,{ }^{* *} \mathrm{P}<0.01$ vs. control.
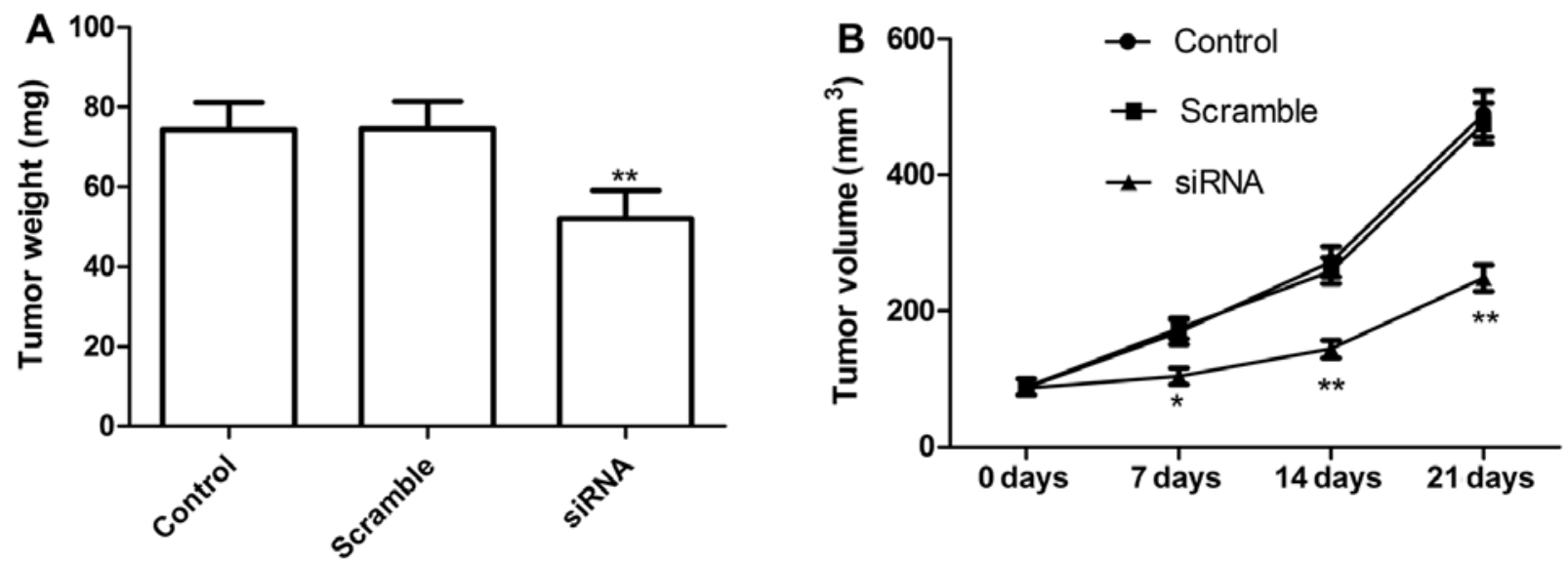

Figure 6. Knockdown of TLR4 inhibits lung tumor growth in vivo. (A) Tumor weight of mice 21 days after treatment with indicated plasmid. (B) Tumor volume of mice at days 7,14 and 21 . Data are expressed as the means $\pm \mathrm{SD}$; ${ }^{*} \mathrm{P}<0.05,{ }^{* *} \mathrm{P}<0.01$ vs. control.

TLR4 knockdown in lung cells inhibits cell migration and cell invasion. We next determined if knockdown of TLR4 could inhibit tumor growth by a xenograft tumor model. At 7 days after the end of treatment, mice were sacrificed and tumor weights were measured. The tumor weight was significantly lower in the knockdown TLR4 group than in control and scramble groups $(\mathrm{P}<0.01$; Fig. $6 \mathrm{~A})$. In addition, tumor volume also was determined at different time. Tumor volume in knockdown TLR4 group groups were significantly diminished when compared with the scramble and control group (Fig. 6B). These data demonstrated that knockdown of TLR4 suppressed tumor growth of NSCLC in vivo.

Effects of TLR4 on PI3K/AKT signaling pathway in A549 cells. To clarify the molecular mechanisms involved due to downregulation of TLR4 inhibition the growth of human lung tumor in vitro and in vivo, we focused on the effects of knockdown of TLR4 on the activation of PI3K/AKT signaling pathway, which participate in the main intracellular signaling pathway required for cell proliferation and survival. As shown

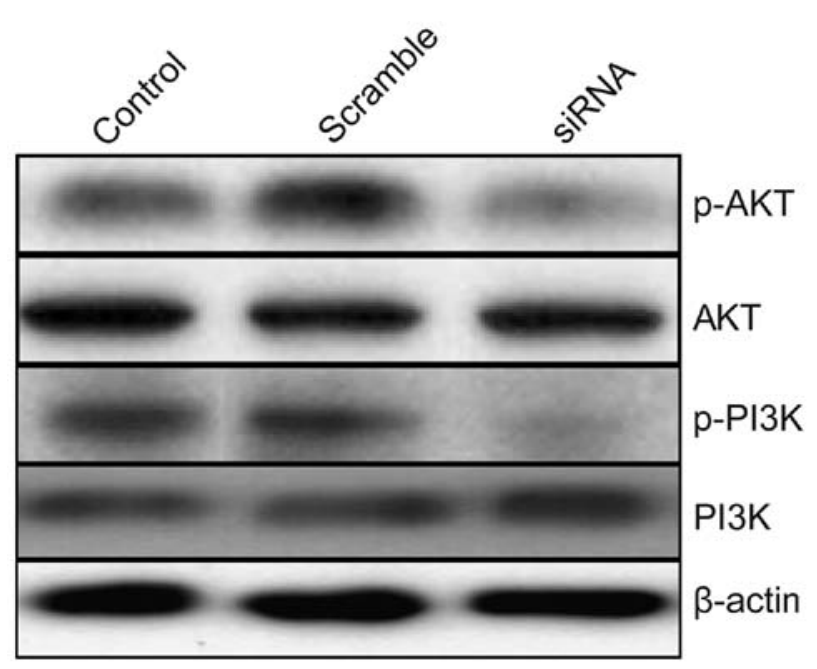

Figure 7. Knockdown of TLR4 inhibits PI3K/AKT signaling in A549 cells. Western blot analysis was performed using specific antibodies against the indicated proteins after treatment with indicated plasmid. Blots were reprobed for $\beta$-actin to normalize each lane for protein content. 
in Fig. 7, compared with control group and scramble group, knockdown of TLR4 by siRNA resulted in a significant suppression of phosphorylation of Akt and PI3K. These results indicate that reduction of TLR4 by siRNA inhibits lung tumor cell growth, to some extent, by suppressing the PI3K/AKT signaling pathway.

\section{Discussion}

Lung cancer is one of the leading causes of cancer-related mortality worldwide (25). Lung cancer mortality rates have been rising in recent decades. It has been shown that chronic inflammatory disease is a risk factor for lung cancer (26). Since TLR-4 is also actively involved in the immune response against cancers, some researchers have suggested that TLR-4 exerts both a defensive role in normal cells and a negative role in cancer cells. However, the available evidence is still not conclusive on the link between TLR- 4 and lung cancer. Bauer et al found that mutated TLR-4 in mice had less lung capillary permeability, less weight loss, leukocyte inflammation, and primary tumor formation (27), and that TLR-4 activation could protect the lungs from being inflamed during any potential tumorigenesis (28). This finding suggests the potential role of TLR-4 for airway inflammation and lung cancer progression. Yet, growing evidence has also found that TLR-4 is constantly expressed and upregulated in human lung cancer cells $(18,20)$. He et al $(18)$ found that the level of TLR-4 was significantly linked with the production of immunosuppressive cytokines, production of proangiogenic chemokine and with resistance to apoptosis by lung cancer cells. Zhang et al (20) showed that TLR4 expression was increased in patients with NSCLC, and TLR4 expression levels correlated with malignancy. In this study, our result showed that TLR-4 expression was elevated in most patients with NSCLC, and its expression level correlated with key pathological characteristics, such as, tumor differentiation, stage and metastasis, which was similar with previous results (20). These results imply that the presence of TLR4 in cancer cells may possess a negative role in lung cancer progression and metastasis.

A study on a larger number showed that TLR4 expression was increased in cancer cell or highly malignant tissues $(20,29,30)$, and that activation of the TLR4 signaling transduction pathway promoted tumor progression and resistance to apoptosis (31). Hua et al showed that upregulation of TLR4 in human prostate cancer cells correlated positively with tumor metastasis (31). Kelly et al found that activation of the TLR4 signaling transduction pathway promoted tumor progression and chemo-resistance of epithelial ovarian cancer cells (11). Similar results were also observed in human head and neck squamous cell carcinoma and breast cell carcinoma where stimulation of TLR4 enhanced (29,32). In addition, growing evidence has shown that knockout TLR4 could inhibit cancer cell proliferation, cell metastasis and induce cancer cell apoptosis $(11,17,29,32)$. Therefore, knocking down TLR4 from cancer cells could reduce tumor metastasis whereas stimulation of TLR4 on cancer cells would enhance the development of aggressive tumors. In the present study, we constructed pcDNA3-CMV vectors expressing two small hairpin siRNA oligonucleotides targeting TLR4 and transfected A549 cells to study the role of TLR4 in lung cancer and found that downregulation of TLR4 expression using RNA silencing approach in A549 tumor cells significantly suppressed cell proliferation, migration and invasion, and induced tumor apoptosis in vitro, and suppressed tumor growth in vivo.

An opinion that chronic inflammation promotes tumor development and progression has been supported by many epidemiological studies and experimental findings $(33,34)$. It has been reported that when tumor cells are stimulated with lipopolysaccharides (LPS), a ligand for TLR4, the proinflammatory factors such as nitric oxide, IL-6 and IL-12 are expected to be released from tumor cells, attracting and activating inflammatory cells. Moreover, these factors play crucial roles in resistance of tumor cells to cytotoxic T lymphocyte (CTL) and natural killer cell (NKC) attack and facilitate evasion from immune surveillance (30). He et al found that knockdown of TLR4 in vitro lead to TLR4-related inflammatory cytokines being markedly depressed and so it could weaken the ability to the resistance of MDA-MB-231 to CTL and NKC attack and facilitate evasion from immune surveillance. In this study, our result showed that downregulation of TLR4 expression significantly decreased TNF- $\alpha$ and IL-6 levels, which was consist with previous results (18). These results may indicate that TLR4 knockdown in vivo inhibited the growth and promoted the death of lung tumors.

It has been reported that TLR-4 is involved in signal pathway regulation. It has been shown that the expression of higher levels of TLR-4 on human prostate adenocarcinoma (DU-145) cells and its activation, lead to NF- $\kappa$ B and proinflammatory cytokine production through the MyD88dependent pathway (35). Another study showed TLR-4/ MyD88-dependent signaling pathway involvement in laryngeal carcinoma progression (36). Hartmann et al showed that activated TLR-4 expression could enhance cancer cell growth, $\mathrm{NF}-\kappa \mathrm{B}$ translocation, and activated phosphatidylinositol 3-kinase/Akt pathway (37). Furthermore, He et al found that TLR4 activation contributes to active p38MAPK pathway, which is necessary for increased VEGF and IL-8 production (18). Hua et al study revealed evidence of a multifaceted signaling network operating downstream of TLR4-mediated tumor cell invasion, proliferation and survival (31). The phosphatidylinositol 3-kinase (PI3K)/AKT signaling pathway plays an important role in survival when cells are exposed to various kinds of apoptotic stimuli (38). Recent reports have indicated that the activation of Akt pathway is implicated in conferring resistance to conventional chemotherapy and multiple chemotherapeutic agents on cancer cells (39). Therefore, in the present study, we mainly focus on effect of TLR4 on the PI3K/AKT signaling pathway by western blot assay. Our results showed that downregulation of TLR4 expression using RNA silencing suppressed phosphorylation of Akt and PI3K, which indicate that TLR4 silencing inhibits tumor cell growth, to some extent, by suppressing activation of the PI3-K/Akt pathway signaling.

In conclusion, the present study demonstrated that TLR4 was elevated in most NSCLC and its expression level correlated with key pathological characteristics including clinical stage and metastasis and that knockdown of TLR4 could actively inhibit proliferation and survival of lung cancer cells in vitro and in vivo. Taken together, our results suggest RNAi-directed targeting of TLR4 may be a beneficial strategy for lung cancer therapy. 


\section{Acknowledgements}

This study was supported by Science and Technology Research and Innovation Team funded of Jilin province (JL2013018).

\section{References}

1. Jemal A, Siegel R, Ward E, Hao Y, Xu J and Thun MJ: Cancer statistics, 2009. CA Cancer J Clin 59: 225-249, 2009.

2. Reungwetwattana T, Weroha SJ and Molina JR: Oncogenic pathways, molecularly targeted therapies, and highlighted clinical trials in non-small-cell lung cancer (NSCLC). Clin Lung Cancer 13: 252-266, 2012.

3. Schiller JH: Small cell lung cancer: defining a role for emerging platinum drugs. Oncology 63: 105-114, 2002.

4. Roy M, Luo YH, Ye M and Liu J: Nonsmall cell lung cancer therapy: insight into multitargeted small-molecule growth factor receptor inhibitors. Biomed Res Int 2013: 964743, 2013.

5. Medzhitov R, Preston-Hurlburt P and Janeway CA Jr: A human homologue of the Drosophila Toll protein signals activation of adaptive immunity. Nature 388: 394-397, 1997.

6. Takeda K, Kaisho T and Akira S: Toll-like receptors. Annu Rev Immunol 21: 335-376, 2003.

7. Medzhitov R and Janeway CA Jr: Decoding the patterns of self and nonself by the innate immune system. Science 296 298-300, 2002

8. Elson G, Dunn-Siegrist I, Daubeuf B and Pugin J: Contribution of Toll-like receptors to the innate immune response to Gram-negative and Gram-positive bacteria. Blood 109: $1574-1583,2007$.

9. De Bouteiller O, Merck E, Hasan UA, et al: Recognition of double-stranded RNA by human toll-like receptor 3 and downstream receptor signaling requires multimerization and an acidic pH. J Biol Chem 280: 38133-38145, 2005.

10. Goto Y, Arigami T, Kitago M, et al: Activation of Toll-like receptors 2,3 , and 4 on human melanoma cells induces inflammatory factors. Mol Cancer Ther 7: 3642-3653, 2008.

11. Kelly MG, Alvero AB, Chen R, et al: TLR-4 signaling promotes tumor growth and paclitaxel chemoresistance in ovarian cancer Cancer Res 66: 3859-3868, 2006.

12. Fukata M, Chen A, Vamadevan AS, et al: Toll-like receptor-4 promotes the development of colitis-associated colorectal tumors. Gastroenterology 133: 1869-1881, 2007.

13. O'Neill LA, Bryant CE and Doyle SL: Therapeutic targeting of Toll-like receptors for infectious and inflammatory diseases and cancer. Pharmacol Rev 61: 177-197, 2009.

14. $\mathrm{Yu} \mathrm{L}$ and Chen S: Toll-like receptors expressed in tumor cells: targets for therapy. Cancer Immunol Immunother 57: 1271-1278, 2008.

15. Garza-Gonzalez E, Bosques-Padilla FJ, Mendoza-Ibarra SI, Flores-Gutierrez JP, Maldonado-Garza HJ and Perez-Perez GI: Assessment of the toll-like receptor 4 Asp299Gly, Thr399Ile and interleukin-8 -251 polymorphisms in the risk for the development of distal gastric cancer. BMC Cancer 7: 70 , 2007.

16. Wang JP, Zhang Y, Wei X, et al: Circulating Toll-like receptor (TLR) 2, TLR4, and regulatory $\mathrm{T}$ cells in patients with chronic hepatitis C. APMIS 118: 261-270, 2010

17. Yang H, Zhou H, Feng P, et al: Reduced expression of Toll-like receptor 4 inhibits human breast cancer cells proliferation and inflammatory cytokines secretion. J Exp Clin Cancer Res 29: 92, 2010.

18. He W, Liu Q, Wang L, Chen W, Li N and Cao X: TLR4 signaling promotes immune escape of human lung cancer cells by inducing immunosuppressive cytokines and apoptosis resistance. Mol Immunol 44: 2850-2859, 2007.

19. Hiratsuka S, Watanabe A, Sakurai Y, et al: The S100A8-serum amyloid A3-TLR4 paracrine cascade establishes a pre-metastatic phase. Nat Cell Biol 10: 1349-1355, 2008.
20. Zhang YB, He FL, Fang M, et al: Increased expression of Toll-like receptors 4 and 9 in human lung cancer. Mol Biol Rep 36: 1475-1481, 2009.

21. Livak KJ and Schmittgen TD: Analysis of relative gene expression data using real-time quantitative PCR and the 2(-Delta Delta C(T)) method. Methods 25: 402-408, 2001.

22. Campo E, Merino MJ, Liotta L, Neumann R and StetlerStevenson W: Distribution of 72KD type IV collagenase in nonneoplastic and neoplastic thyroid tissue. Hum Pathol 23: 1395-1401,1992.

23. Lowry OH, Rosebrough NJ, Farr AL and Randall RJ: Protein measurement with the Folin phenol reagent. J Biol Chem 193: 265-275, 1951.

24. Zhang D, Chen ZG, Liu SH, et al: Galectin-3 gene silencing inhibits migration and invasion of human tongue cancer cells in vitro via downregulating beta-catenin. Acta Pharmacol Sin 34: 176-184, 2013.

25. Mendez M, Custodio A and Provencio M: New molecular targeted therapies for advanced non-small-cell lung cancer. J Thorac Dis 3: 30-56, 2011.

26. Yao H and Rahman I: Current concepts on the role of inflammation in COPD and lung cancer. Curr Opin Pharmacol 9: 375-383, 2009.

27. Bauer AK, Dixon D, DeGraff LM, et al: Toll-like receptor 4 in butylated hydroxytoluene-induced mouse pulmonary inflammation and tumorigenesis. J Natl Cancer Inst 97: 1778-1781, 2005.

28. Bauer AK, Fostel J, Degraff LM, et al: Transcriptomic analysis of pathways regulated by toll-like receptor 4 in a murine model of chronic pulmonary inflammation and carcinogenesis. Mol Cancer 8: 107, 2009

29. Szczepanski MJ, Czystowska M, Szajnik M, et al: Triggering of Toll-like receptor 4 expressed on human head and neck squamous cell carcinoma promotes tumor development and protects the tumor from immune attack. Cancer Res 69: 3105-3113, 2009.

30. Huang B, Zhao J, Li H, et al: Toll-like receptors on tumor cells facilitate evasion of immune surveillance. Cancer Res 65: 5009-5014, 2005

31. Hua D, Liu MY, Cheng ZD, et al: Small interfering RNA-directed targeting of Toll-like receptor 4 inhibits human prostate cancer cell invasion, survival, and tumorigenicity. Mol Immunol 46: 2876-2884, 2009.

32. Ahmed A, Wang JH and Redmond HP: Silencing of TLR4 increases tumor progression and lung metastasis in a murine model of breast cancer. Ann Surg Oncol 20 (Suppl) 3: S389-S396, 2013.

33. Coussens LM and Werb Z: Inflammation and cancer. Nature 420: 860-867, 2002

34. Kusmartsev S and Gabrilovich DI: Immature myeloid cells and cancer-associated immune suppression. Cancer Immunol Immunother 51: 293-298, 2002.

35. Gatti G, Quintar AA, Andreani V, et al: Expression of Toll-like receptor 4 in the prostate gland and its association with the severity of prostate cancer. Prostate 69: 1387-1397, 2009.

36. Starska K, Forma E, Brys M, et al: The expression of TLR pathway molecules in peripheral blood mononuclear cells and their relationship with tumor invasion and cytokine secretion in laryngeal carcinoma. Adv Med Sci 57: 124-135, 2012.

37. Hartmann E, Wollenberg B, Rothenfusser S, et al: Identification and functional analysis of tumor-infiltrating plasmacytoid dendritic cells in head and neck cancer. Cancer Res 63: 6478-6487, 2003

38. Oka N, Tanimoto S, Taue R, et al: Role of phosphatidylinositol-3 kinase/Akt pathway in bladder cancer cell apoptosis induced by tumor necrosis factor-related apoptosis-inducing ligand. Cancer Sci 97: 1093-1098, 2006.

39. Kai K, D'Costa S, Sills RC and Kim Y: Inhibition of the insulin-like growth factor 1 receptor pathway enhances the antitumor effect of cisplatin in human malignant mesothelioma cell lines. Cancer Lett 278: 49-55, 2009. 